\title{
Induction of Hypometabolism as a Novel Strategy to Minimize Ischemia Reperfusion Injury in Renal Reconstruction Surgeries
}

\section{Bobby Alexander ${ }^{1}$, Meris James ${ }^{2}$, Andrew Fishman ${ }^{1}$, Michael Grasso ${ }^{1}$ and Thambi Dorai ${ }^{1^{*}}$}

${ }^{1}$ Department of Urology, New York Medical College, USA

${ }^{2}$ Department of Pharmacology, New York Medical College, USA

"Corresponding author: Thambi Dorai, Department of Urology, New York Medical College, 15 Dana Road Basic Science Building, BSB-12910954 Valhalla, NY 10595, USA, Tel: 001-845 558 2751; E-mail: thambi_dorai@nymc.edu

Received date: May 27, 2016; Accepted date: June 16, 2016; Published date: June 23, 2016

Copyright: (c) 2016 Alexander B, et al. This is an open-access article distributed under the terms of the Creative Commons Attribution License, which permits unrestricted use, distribution and reproduction in any medium, provided the original author and source are credited.

\begin{abstract}
Purpose: Hibernation is an altered physiological state which is marked by a characteristic lowering of body temperature and extreme metabolic rate depression. During this period, an animal undergoing hibernation may have its heart rate and blood flow decrease to $1 / 30$ and its oxygen consumption to $1 / 100$ of its respective euthermic levels. Even though these physiological parameters mimic conditions of ischemia their organs particularly their brain, heart, liver and kidneys do not show any detrimental effects of severely reduced blood flow when the animal comes out of hibernation. We wished to explore the benefits of inducing such a hypometabolic state in a mouse ischemiareperfusion injury model.
\end{abstract}

Methods: We investigated the pre-conditioning effect of administering 5'-Adenosyl Monophosphate (5'-AMP) to mice to create a state of hypometabolism akin to hibernation. This hibernation-like state is used to study its protective effect on a subsequent renal ischemic episode.

Results: Our results strongly suggest that an induction of a hypometabolic state greatly attenuates the ischemic and reperfusion damage to the kidneys, as studied by several specific parameters.

Conclusions: We conclude that reprogramming of the kidney metabolism to a hypometabolic state before ischemia may protect the organ from oxidative damage and reduce ischemia/reperfusion injury. This concept may also be applicable to improve clinical outcomes in renal reconstruction as well as transplantation surgeries.

Keywords: Ischemia-reperfusion injury; Hypometabolism; Partial nephrectomy; Warm ischemia; 5'-Adenosine monophosphate

\section{Introduction}

Living organisms are faced with a wide variety of internal and external stressors throughout their life cycle, forcing hem respond in ways by which they can continue to sustain life. These responses involve adjustments in cellular metabolism to meet new demands at the same time striving to maintain a balance between cellular processes. In cases of extreme heat/cold, lack of oxygen, dehydration, food deprivation, certain organisms go through hypometabolism as a survival mechanism [1-3]. The entrance into a hypometabolic state/ torpor is the body's attempt to minimize energy expenditure and to conserve energy under metabolic stress caused by an insufficient nutrient supply. In certain animals hypometabolic states are integral parts of phenomena including hibernation, estivation, diapause and anaerobiosis [1-4]. During the winter season, metabolic rates of the hibernators such as bears and ground squirrels are suppressed by over $90 \%$ and their core body temperatures can potentially reach a few degrees just above the ambient temperature or can go as low as $5^{\circ} \mathrm{C}$ [1-5]. Cellular processes such as DNA replication, transcription, as well as protein synthesis are largely suppressed during this period. The adenosine 5' triphosphate (ATP) production from glucose is reduced and lipids serve as the major substrates for meeting energy requirements [1-5]. However, the uniqueness about hibernators is that these changes are rapidly reverted to normal metabolism during their arousal period (whether daily or seasonal) without any apparent signs of cellular or organ injury. Non-hibernating mammals experience slight fluctuations in metabolic rate over the course of their daily sleep and awake cycles. During their sleep/resting state, the metabolic rate of most mammals decreases by about $20 \%$ below the level of metabolic rate during their awake/active state. This accounts for only a $0.5-2^{\circ} \mathrm{C}$ decrease in their core body temperature compared to the drastic reductions in body temperatures of true hibernators [6]. Unlike hibernators, non-hibernating mammals are not engineered to immediately enter hypometabolism under extreme environmental conditions. On the contrary, under cold temperatures, the metabolic rate of non-hibernators increases due to thermoregulatory heat production to keep the body temperature at $37^{\circ} \mathrm{C}$ [6]. True endotherms (i.e. humans) maintain their physiological parameters at a constant level over a wide variation in environmental temperature. Maintenance of body temperature at a very narrow range, around $98.6^{\circ} \mathrm{F}$ or $37^{\circ} \mathrm{C}$ is critical for normal human body functions. However, this tight regulation of thermal stability and various other physiological factors in humans and most endotherms comes at an enormous cost of energy [1-5]. As a result, survival of non-hibernators requires a continuous high supply of food and energy [6]. On the other hand, hibernators spend days or weeks in a state of torpor, where all their physiological functions are profoundly suppressed, their organs are severely hypoperfused and their metabolic rate falls to less than $5 \%$ of the euthermic resting state [1-6]. Yet, when these animals emerge from 
their hibernation state, their brain, kidneys, lungs and their heart spring back to normalcy almost immediately. Therefore, the mechanisms of induction of a hypometabolic state in these hibernators (and in some non-hibernators) and the mechanisms by which they preserve their organs in spite of a severely restricted blood flow have been under great investigation, not only for their unique nature, but also for translational benefits, particularly for their potential cytoprotective role in the clinical situations like myocardial or renal ischemia or stroke [7-10]. Also, application of the concepts of hypometabolism may also offer great potential benefit to the field of renal transplantation where the preservation of the integrity of the donor kidney is of paramount importance $[11,12]$. Earlier studies have focused on the induction of a hypometabolic state by the administration of hydrogen sulfide through gaseous or chemical means [13-16]. The main drawback of this approach is that this gasotransmitter (like carbon monoxide) is still considered a cellular poison. It has beneficial effects in attenuating renal ischemia reperfusion injury at extremely low concentrations. But, it has serious toxic effects at higher concentrations highlighting its narrow therapeutic window which is difficult to control in clinical situations, particularly after the realization that typical $\mathrm{H}_{2} \mathrm{~S}$ donors such as sodium sulfide and sodium hydrosulfide $\left(\mathrm{Na}_{2} \mathrm{~S}\right.$ and $\left.\mathrm{NaHS}\right)$ are short acting and do not give a continuous release of $\mathrm{H}_{2} \mathrm{~S}$ [17]. In the search for a safe and effective alternative approach to inducing a hypometabolic state, we came across the published studies of Lee and his group [8,9,18-20] which focused on the induction of a hypometabolic state by the administration of adenosine monophosphate (5'-AMP) which is present and metabolically used in every cell of the body. Here, we describe the beneficial effects of administration of 5'-AMP in protecting the kidney in mice after a renal ischemic episode and suggest that this could be a safe and non-toxic way to preserve the global parenchymal function of the kidney in clinical situations such as renal reconstruction surgeries and partial nephrectomies where temporary renal artery occlusion is warranted. Our earlier studies have described several approaches to extending the warm ischemia time in partial nephrectomy procedures [21-25]. Induction of hypometabolism could be yet another method by which renal ischemia and reperfusion injury could be minimized, ultimately benefitting the patient. Our results strongly support a hypothesis that the activation of the enzyme AMP-activated kinase (AMPK) in the kidney may play an important role in transmitting the hypometabolic signals that would mitigate the effects of renal ischemia-reperfusion injury.

\section{Materials and Methods}

\section{Animals}

Female C57BL/6 mice (10-12 weeks of age, weighing between 20 and 25 grams) were acquired from Charles River Laboratories and acclimatized to the conditions in the animal research facility. All procedures were performed in accordance with the established protocols of the Institutional Animal Care and Use Committee (IACUC) of the Institute of Comparative Medicine at the New York Medical College. Mice were maintained in the facility with a standard 12 hour dark/light cycle and they had access to food and water ad libitum. At the end of the acclimation period, the mice were randomized into 4 groups of 8 animals: 1) "sham" group 2) "ischemia only" group 3) "AMP -only" group and 4) "AMP + ischemia" group. Power analysis was performed for arriving at the number of animals selected for each group. Statistical variations were analyzed by using the two-way Anova test whenever necessary.

\section{Induction of hypometabolism/hypothermia in experimental animals}

The administration of 5'-Adenosine monophosphate (5'-AMP) was essentially the same as that described by Daniels et al [19]. Briefly, each mouse in the AMP only group received intraperitoneally a solution (in phosphate buffered saline, PBS) of 5'-AMP (Sigma, \# A-1752). The animals entered in to a hypometabolic state almost immediately with little or no physical activity. After AMP administration, the animals were kept in a climate controlled chamber maintained at $15^{\circ} \mathrm{C}$ for a period of 5 hours. Under these conditions, their heart rates were reduced to less than $10 \%$ of their normal rates at $37^{\circ} \mathrm{C}$. The core body temperature (CBT) was followed for a period of 5 hours after AMP administration with the help of a rectal probe thermometer (Bioseb Inc, Type KJT). The CBT dropped close to the temperature of the climate controlled chamber and was maintained for nearly 4 hours. Their $\mathrm{O}_{2}$ consumption rate was followed under these conditions using an Oxy Max test chamber system equipped with $\mathrm{CO}_{2}$ and $\mathrm{O}_{2}$ sensors (Columbus Instruments Inc, Columbus, $\mathrm{OH}$ ). As described in $[18,19]$ the reverse flip behavior, i.e., their ability to right themselves on their feet was used to determine their arousal from this state of metabolic depression, which was roughly 240 minutes after the 5'-AMP injection. After the animals exhibit the reverse flip behavior, they warmed up and resumed normal mobility thereafter. After the animals recover from this hypometabolic state, they were returned to their animal husbandry cages kept at room temperature $\left(25^{\circ} \mathrm{C}\right)$. This protocol for hypometabolic pre-conditioning was repeated on days 3,5 and 7.

\section{Left renal ischemia}

Since the administration of general anesthesia (by a ketamine, xylazine mixture) amplify the effect of AMP induced hypometabolism and the generation of AMP-induced hypometabolism slows down the body's capacity to metabolize/clear the anesthetics, isofluorane induced general anesthesia was given to animals just before surgery on day 9 , according to the protocols established in the animal facility (Zhao Z, personal communication). The animals that had been injected (i.p) with a fresh dose of 5'AMP were subjected to left renal ischemia, induced by clamping the renal pedicle for 30,45 and 60 minutes essentially according to the procedure described earlier [21]. After a reperfusion period of 24 hours, the animals were sacrificed. Their ischemic and contra-lateral kidneys were harvested and processed for both immunohistochemistry and protein chemistry analyses. Sham operated animals were not subjected to 5-AMP treatment but were processed through a mock procedure without the actual renal artery occlusion. The "AMP-only" groups of animals were subjected to AMP-preconditioning protocol only without renal ischemia. The "ischemia only" groups of animals were subjected to the left renal ischemia procedure only without any pre-conditioning by AMP treatment. Finally, the "AMP + Ischemia" group received both AMP-mediated pre-conditioning and left renal ischemia for the above mentioned periods.

\section{Histology and immunohistochemistry}

The harvested kidney specimens were subjected to pathological examination essentially as described earlier by our laboratory $[21,24,25]$. Briefly, specimens were fixed in $10 \%$ buffered formalin for 24 hours and transferred to $70 \%$ ethanol. After embedding in paraffin, 
$4 \mu \mathrm{m}$ thick sections were made and stained with hematoxylin and eosin (H\&E) using standard procedures. A set of parallel sections were processed for Periodic Acid Schiff (PAS) staining. Medullary tubular necrosis was assessed and scored as described earlier [24,25]. For Immunohistochemical detection of oxidative stress in paraffin fixed sections of the kidney in the experimental groups, a commercially available kit was used (OxyIHC oxidative stress detection kit, Millipore Corporation, Billerica, MA) following the procedures prescribed by the manufacturer.

\section{Estimation of serum creatinine in the animal groups}

This was done essentially as described earlier using a commercially available calorimetric kit (Cayman Chemicals, Ann Arbor, MI) $[24,25]$. As an independent parameter, serum cystatin- $C$ levels were also followed (see below).

\section{Immunoblot analysis}

The protein analysis procedures were done exactly as described earlier $[24,25]$. Serum specimens were recovered from the animals at end point for the various groups as described in Figure 1. Twenty five microliters of the serum specimens were subjected to $15 \%$ denaturing gel electrophoresis, blotted onto nitrocellulose membranes and probed with a commercially available cystatin-C antibody (Cell Signal Inc, Danvers, MA). Similarly, immunoblot analysis was performed using $12 \%$ denaturing gels on normalized protein extracts made from various specimens collected with blocking by $5 \%$ milk (Blotto, Santa Cruz, Inc) in Tris Buffered Saline (TBS) containing 0.05\% Tween 20. Protein estimations were performed using the BCA assay kit (Pierce/ ThermoFisher, Inc) after removing the interfering substances using the Compat-Able protein assay preparation reagent (Pierce/ThermoFisher, Inc).

Electrophoresced proteins on the blots were probed with antibodies for galectin-3 (mouse monoclonal, Santa Cruz, Inc); Mucin-1 (mouse monoclonal, Santa Cruz, Inc); Neutrophil associated gelatinase-2 (NGAL, rabbit polyclonal, Santa Cruz, Inc); AMPK (rabbit polyclonal, Santa Cruz, Inc); phospho-AMPK (pThr172, rabbit polyclonal, Santa Cruz, Inc); Acetyl Coenzyme A Carboxylase (ACC, Abcam Inc, rabbit polyclonal); phospho-ACC (pS79, Abcam Inc,rabbit polyclonal); Nrf2 (Cell Signal Inc, rabbit polyclonal), Hypoxia inducible factor-1 alpha (HIF-1a, Novus Biologicals, rabbit polyclonal). Proteins loading on the gels were routinely monitored by probing identical blots for the expression of mouse $\beta$-actin (mouse monoclonal, Santa Cruz, Inc).

\section{Results}

Our initial studies focused on the generation of the hypometabolic state and characterization of the torpor-like parameters in mice in the laboratory under the conditions described in Materials and Methods. Immediately after the injection of 5 -AMP the animals went into torpor-like state, suggesting that 5'-AMP could mimic a state of metabolic suppression seen in hibernating animals. After the injection of 5'-AMP, the animals were maintained for next 5 hours in a cooled chamber with an ambient temperature of $15^{\circ} \mathrm{C}$. We also noticed that cooling down the animal to near $15^{\circ} \mathrm{C}$ produced a better and deeper torpor and a "suspended animation" state in these animals, a feature confirmed by earlier studies $[18,19]$. The kinetic parameters for the establishment and the duration of this state were measured by following their core body temperature as well as their oxygen consumption rate, which are described in Figure $1 \mathrm{~A}$ and $1 \mathrm{~B}$ respectively. Examination of both these parameters revealed a triphasic curve. First, there is a sudden drop in core body temperature (CBT) as well as in the oxygen consumption rate $\left(\mathrm{VO}_{2}\right)$. In this entrance phase, CBT dropped to near $15^{\circ} \mathrm{C}$ and the $\mathrm{VO}_{2}$ dropped to around $20 \mathrm{ml} / \mathrm{kg} /$ min. In the second maintenance phase, $\mathrm{CBT}$ was maintained near $15^{\circ} \mathrm{C}$ for the next 3 hours and the $\mathrm{VO}_{2}$ was maintained for the next 2 hours as shown in Figure 1A and 1B. During these two states, their heart rate was nearly $5 \%$ of their normal rate, as measured by a heart rate monitor (data not shown). Finally, there is an arousal phase where the $\mathrm{CBT}$ as well as $\mathrm{VO}_{2}$ come back to near normal as seen in the untreated control mice. At the end of this arousal period, the animal is able to right itself when laid on its back (reverse flip behavior). When the animals are fully recovered, they were transferred to their animal husbandry cages. This procedure was repeated on days 3, 5 and 7, designed as a "pre-conditioning" method to prepare the animals for an episode renal ischemia. On day 9, the animals were injected with 5'AMP at the same dose and allowed to enter into the state of deep hypometabolism. The kinetics of the lowering of the core body temperature, oxygen consumption rate and the heart rate suggested that there is a "window of opportunity" of around 2 hours where the blood flow to the kidney is very likely severely reduced to a "nearischemic" state when a renal artery occlusion procedure could be performed without any damaging effect on the kidney. However, a systematic renal blood flow measurements were not done in this study. We reasoned that the repeated production of this "near ischemic" state is similar to the "ischemic pre-conditioning" perfected by other investigators [26,27]. Left renal ischemia for defined periods of time was done as described in Figure 2 and the animals were returned to their cages to start a reperfusion period of 24 hours as described in the protocol. At end point the animals were sacrificed and the ischemic as well as the contra-lateral kidneys were harvested and processed for further analysis as described below. In parallel, serum samples were also prepared from these animals just before renal artery occlusion as well as at the end point for the analysis of their creatinine as well as Cystatin-c levels.

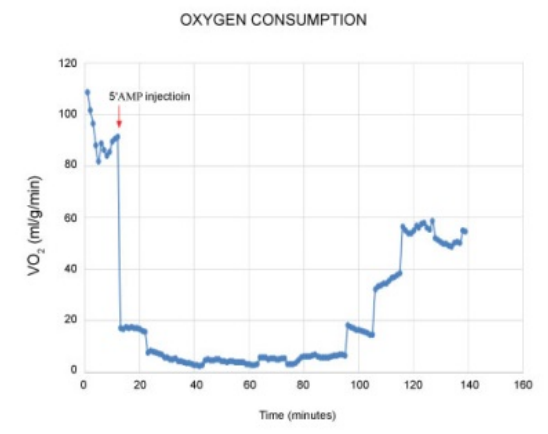

Figure 1: A. Tracing of the core body temperature (CBT) in a representative mouse (red diamonds) that was treated with 5'-AMP (see Materials and Methods) and a control mouse (blue circles). Both mice were kept at an ambient temperature of $15^{\circ} \mathrm{C}$ for the duration of the experiment. The temperature measurements were taken with the help of a mouse rectal thermometer. 


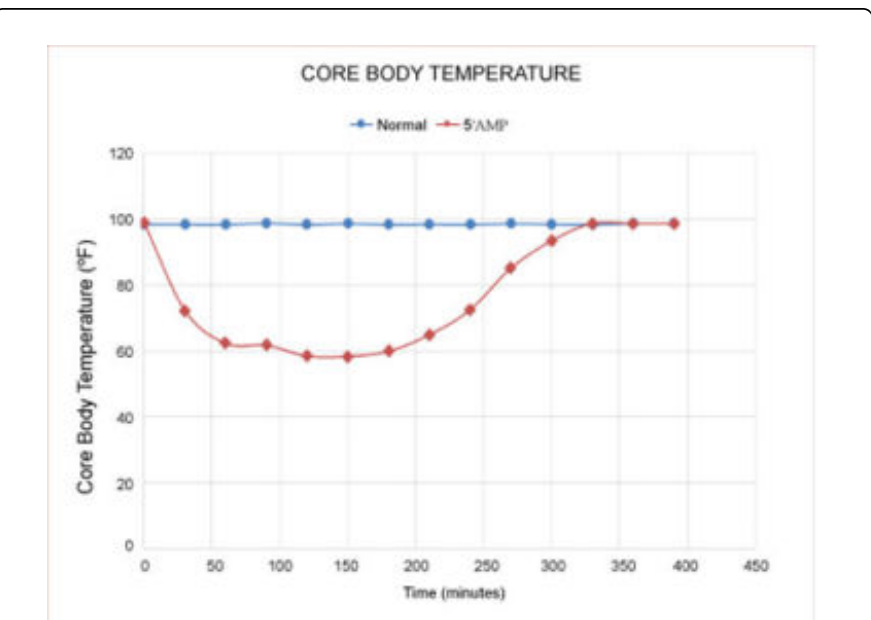

Figure 1: B. Measurement of the oxygen consumption rate of a mouse that was treated with 5'-AMP (blue line) and a control mouse that was not treated with 5'AMP (red line) using a OxyMax test chamber. Oxygen consumption rate $\left(\mathrm{VO}_{2}\right)$ was expressed as milliliters consumed per $\mathrm{kg}$ per minute. In this representative mouse recording, the initial decline in the oxygen consumption just before 5'AMP injection was very likely caused by stress while there is a characteristic and precipitous drop in oxygen consumption immediately afterwards.

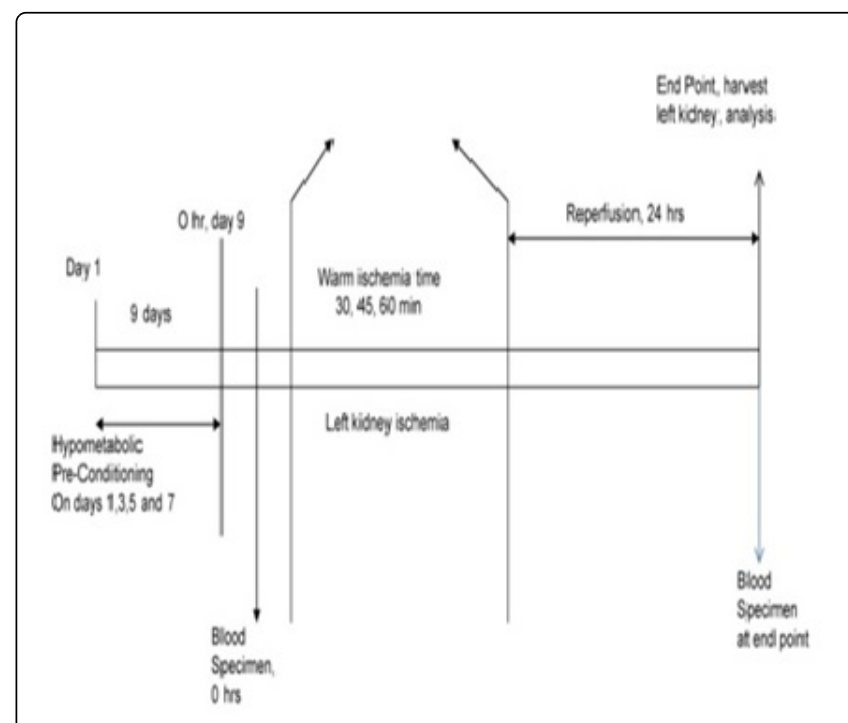

Figure 2: Design of the animal protocol.

Histology and immunohistochemistry analyses confirmed our hypothesis that pre-conditioning by repeated induction of hypometabolism with a subsequent renal ischemic episode produced significantly less renal damage compared to the respective controls, as shown in Figures 3-5. Figure 3 shows the H\&E analysis of the tubular damage in the kidney in sham (top left panel), AMP only (top right panel), ischemia only (bottom left panel) and AMP + ischemia (bottom right) treated animals. Only the pattern seen after a 45 minute renal ischemia is shown for comparative analysis. Similar to sham kidney, the "AMP only" kidney shows negligible kidney tubular damage. Ischemia only group showed typical tubular damage pattern, which included severe tubular epithelial degeneration, necrosis and frequent luminal clogging. Whereas, the AMP + ischemia group showed significantly less tubular damage compared to the ischemia only group. Figure 4 shows the Periodic Acid Schiff staining of the adjacent sections of the kidneys from various groups. This stain distinguishes the brush border of the renal tubules. As compared to the sham or AMP only kidneys, the ischemia only kidney shows epithelial cell degeneration with a significant loss of the brush border which no longer lines the renal tubules and accumulates in the lumen with cast formation in many areas. Characteristically, the AMP + ischemia group showed less brush border damage in the PAS stain with significantly less degenerative changes described above. The acute tubular necrosis was scored exactly as mentioned earlier $[24,25]$. The values were $3.1+/-0.4,4.0+/-0.5,4.7+/-0.3$ and $3.0+/-0.3$ for ischemia only for 30,45 and 60 minutes followed by AMP with 45 minute ischemia respectively as evaluated by a two -tailed Student $t$ test with a $P$ value of $<0.05$. This again shows that there is a significant reduction in the acute tubular damage when the kidneys were preconditioned by AMP treatment. Thus, these results suggest that a "metabolic suppression pre-conditioning" of the kidneys by treatment with 5'-AMP may be a good method to preserve the kidneys and protect them from severe ischemic damage when a subsequent ischemic episode occurs. If the protective effect of 5'-AMP is functioning in the ischemic kidneys, we postulated that there would be relatively less oxidative stress in the AMP + ischemia group compared to the other control groups. The result of the oxidative stress analysis is shown in Figure 5. This analysis detects the nitrotyrosylated proteins in the paraffin embedded sections of stressed tissues [due to the production of reactive oxygen species (ROS)] immunohistochemically using antibodies against nitrotyrosine. As shown in this Figure there is relatively little or no oxidative stress in the kidneys of the sham or AMP only group. There is an intense ROS staining in the ischemia only group, while the same stain is greatly attenuated in the AMP + ischemia group. This analysis is qualitative proof that metabolic suppression and induction of hypometabolism and a torpor like state conditions the kidney to reduced blood flow and prepares/protects the organ against a subsequent ischemic damage. Similar protection against ischemic damage of the kidney was shown by Bos et al. when they induced a hypometabolic state by the treatment with a hydrogen sulfide $\left(\mathrm{H}_{2} \mathrm{~S}\right)$ [15].

We next performed an extensive analysis of the ischemia specific marker proteins for the various groups of animals and the results are shown in the Figure 6. We followed the levels of three well known renal ischemia markers namely galectin-3 [28], mucin-1 [29] and neutrophil gelatinase associated lipocalin-2 (NGAL) [30] using commercially available antibodies. While the level of expression of these proteins are at the base level for the sham group and AMP only group (lanes 1 and 2) they are significantly up regulated in the ischemia group when the animals are subjected to 30,45 and 60 minute ischemia (lanes 3,4 and 5). Significantly, there is a characteristic down regulation of these proteins when these animals are pre-conditioned with AMP mediated hypometabolism and then subjected to ischemia for the time points mentioned (lanes 6, 7 and 8). Next, we followed the expression of a more reliable ischemia marker in the serum, namely cystatin-C on Immunoblots which again showed significant attenuation of its levels 
Citation: $\quad$ Alexander B, James M, Fishman A, Grasso M, Dorai T (2016) Induction of Hypometabolism as a Novel Strategy to Minimize Ischemia Reperfusion Injury in Renal Reconstruction Surgeries. J Transplant Technol Res 6: 164. doi:10.4172/2161-0991.1000164

Page 5 of 9

when the animals are pre-conditioned before the ischemic episode as shown in Figure 6 [31].

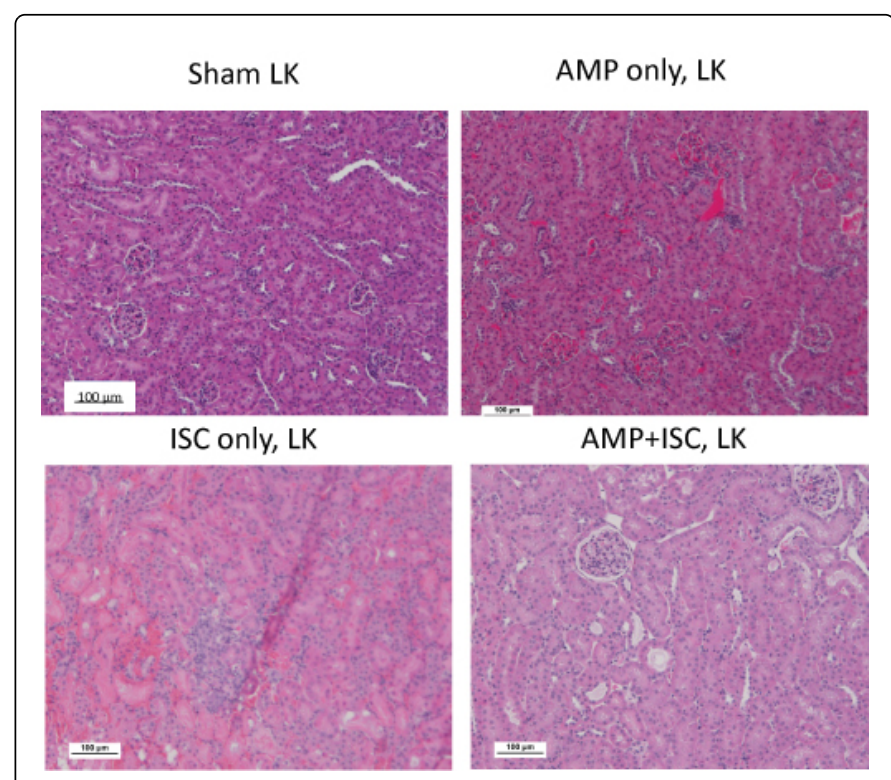

Figure 3: H\&E analysis of the renal tubular damage in the sham operated controls as well as the 3 experimental groups as designed in the animal protocol. LK: left kidney; AMP: 5'-Adenosine monophosphate treated; ISC: ischemia treated; AMP + ISC: pretreatment with AMP followed by ischemia. The H\&E patterns are representative of the animals that faced ischemia for 45 minutes. Scale bars are indicated in each picture.

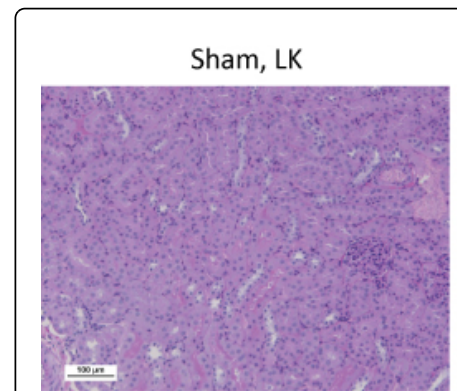

ISC only, LK

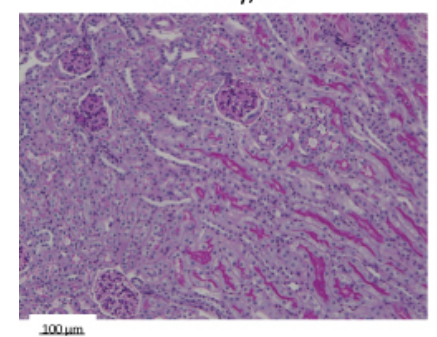

100 um

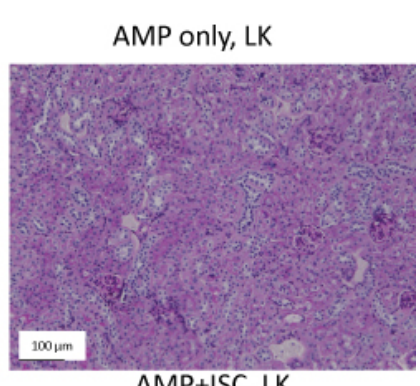

AMP+ISC, LK

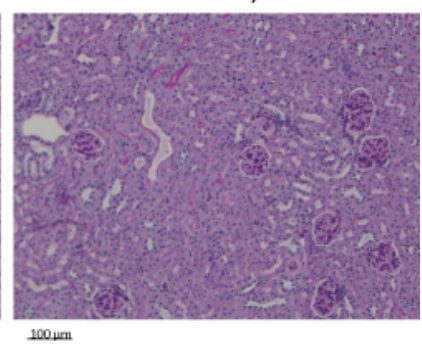

Figure 4: Periodic-Acid Schiff (PAS) analysis of the various animal groups used in this study, with an ischemic period of 45 minutes.

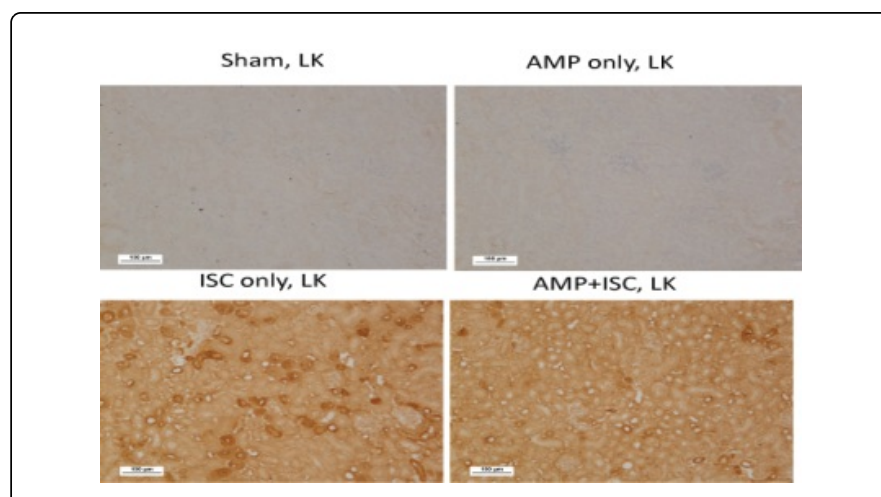

Figure 5: Immunohistochemical analysis of the oxidative stress that occurred in the 4 groups of animals. The ischemia time was for 45 minutes, as shown in Figures 3 and 4. The reactive oxygen species (ROS) were qualitatively analyzed using the OxyIHC kit (Millipore) as described by the manufacturer. Scale bars are as indicated.

As an independent parameter for confirmation of acute tubular necrosis, we also followed the serum creatinine levels using a classical calorimetric assay method, which is shown in Table 1 . The results of serum creatinine assays and the serum cystatin- $C$ levels are in concordance with each other and we conclude that there is a significant attenuation of necrosis in ischemic animals pre-conditioned with AMP. Since AMP activated protein kinase (AMPK) is the target of 5'-AMP, we followed the expression of AMPK as well as its activated form (phospho- AMPK) in the protein extracts made from the various groups mentioned in this study. While the expression of the unphosphorylated version of AMPK is held at a near constant levels, the activated form of AMPK is up regulated in the AMP only as well as the AMP + ischemia groups (lane2 and lanes 6, 7 and 8). These results highly suggest that the protective effect of 5'AMP could be mediated by, at the least in part, by the activation of AMPK by phosphorylation, among other mechanisms. All these protein analyses were done under constant protein loading conditions, as followed by probing for the presence of the house keeping protein $\beta$-actin. Next, we followed the protein expression levels of Acetyl-CoA carboxylase (ACC) as shown in Figure 7. The phosphorylated version of ACC is a functional readout for the activation of AMPK which receives the signal for its activation by 5'-AMP among other agonists [32]. While the level of expression of ACC is nearly constant in sham (panel A, lane 1) AMP-only (panel A, lane 2) ischemia only (panel A, lane 3) and AMP + ischemia (panel A, lane 4) groups there is a an activation of this enzyme in the AMP only (panel B, lane 2) and AMP + ischemia group (panel B, lane 4) when followed by a phospho-specific AMPK antibody. This suggests that the AMPK activation is functional within the kidney as it helps in the inhibition of fatty acid synthesis by phosphorylating ACC. Since the pre-conditioning protocol described in this study involved periodic and drastic reduction in the blood flow to the kidney, which by itself resembles a minor ischemic episode, we anticipated that would be a stabilization of the hypoxia inducible factor-1a (HIF-1a) within the kidney tissue. As shown in Figure 7, panel C, the expression of HIF-1a is at its base levels in sham group (lane 1) while it is gradually up regulated in AMP only group (lane 2), ischemia only group lane 3 ) and finally in the AMP + ischemia group (lane 4). This shows that the AMP mediated hypometabolism and its renal protection is partly due to a hypoxic stabilization of HIF-1a. HIF-1a can also be stabilized due to the production of reactive oxygen species (ROS) [33]. Finally, since 
AMPK is a master "sensor" of the metabolic stress and ATP deficiency states and it is closely linked to the anti-oxidant response system of the cell which is mediated by the transcription factor nuclear factor erythroid-2 related factor (Nrf2), we followed the expression of this protein in the four animal groups in our study [34-38]. Figure 7 panel $\mathrm{D}$ shows that there is an up regulation of this transcription factor in the AMP only group (lane 2) and the AMP + ischemia group (lane 4) compared to the sham (lane 1) and the ischemia only (lane 3 ) groups. In Figure 7, panel $\mathrm{E}$ shows the expression levels of the protein loading control $\beta$-actin for the various groups. These results suggests that the hypometabolism effect mediated by 5 -AMP is multifactorial that could be orchestrated by several proteins/enzymes affected by the AMP-mediated signaling pathways.

\begin{tabular}{|l|l|}
\hline Treatment & Creatinine levels $\mu \mathrm{mol} / \mathrm{L}$, Mean+/-SEM \\
\hline Sham operation & $35+/-7.3$ \\
\hline Ischemia Only, $30 \mathrm{~min}$ & $112+/-5.3$ \\
\hline Ischemia Only, $45 \mathrm{~min}$ & $140+/-8.3$ \\
\hline Ischemia Only, $60 \mathrm{~min}$ & $184+/-7.2$ \\
\hline AMP only & $38+/-5.7$ \\
\hline AMP + Ischemia, 45 min & $65+/-4.4 \#$ \\
\hline$\#=P<0.05$, SEM: Standard Error of the Mean \\
\hline
\end{tabular}

Table1: Serum creatinine values for the various groups.

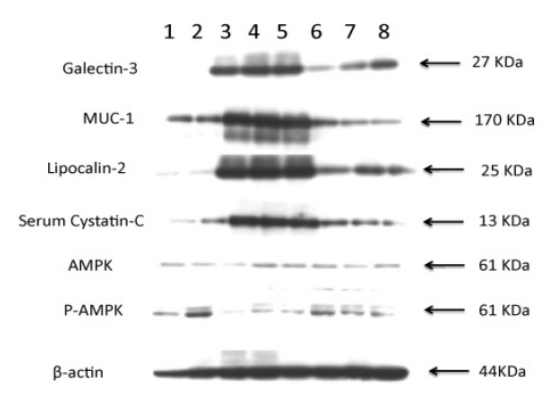

Figure 6: Immunoblot analysis of the various renal ischemia markers in the serum as well as protein extracts made from the kidneys of animals exposed to ischemia for various time points (30, 45 and 60 minutes). See Materials and Methods for the sources of the antibodies used. The molecular weights of the specific ischemia markers (galectin-3, MUC-1, Lipocalin-2 and Cystatin-C are depicted. The levels AMP activated kinase (AMPK) was also analyzed. In parallel, its activation status was also followed using a phospho-specific antibody. For normalization of protein loading, the expression level of $\beta$-actin was followed. Lanes 1: sham operated kidney; lane 2: kidney treated with AMP only. Lanes 3, 4 and 5: kidney that received ischemia only for 30,45 and 60 minutes respectively; Lanes 6,7 and 8: kidney of animals that were preconditioned with AMP and treated with ischemia for 30,45 and 60 minutes respectively.

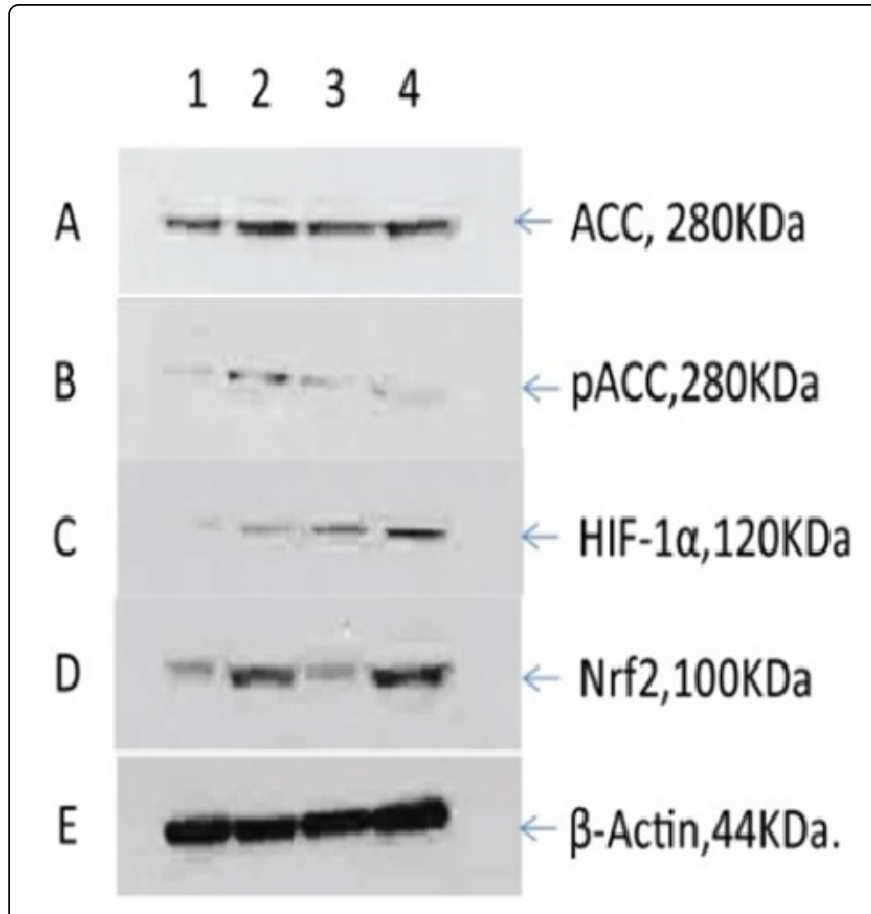

Figure 7: Immunoblot analysis of the protein extracts prepared from sham ( lane 1); 5'-AMP only (lane 2); ischemia only (45 minute episode, lane 3); 5'-AMP preconditioning followed by 45 minute ischemia (lane 4) probed for the expression levels of various ischemia related proteins. Panel A: Acetyl-Coenzyme A Carboxylase (ACC); Panel B: phosphorylated version of ACC; Panel C: expression level of HIF-1a under the experimental conditions used; Panel D: base as well as the up regulated levels of the transcription factor Nrf2; Panel E: level of $\beta$-actin expression followed for loading control. The corresponding molecular weights are indicated.

\section{Discussion}

The studies presented here highlight the remarkable benefit of preconditioning the animal by inducing a hypometabolic state prior to an actual renal ischemic episode. We believe that the pre-conditioning effect is mainly brought up through repeated metabolic suppression similar to what is seen in hibernating animals $[39,40]$.

The metabolic and cell-signaling alterations induced by the administration of 5'-AMP are very likely the reasons for reducing the stress induced by ischemia to the kidney. There are many other ways of inducing a hypometabolic state in experimental animals such as the administration of hydrogen sulfide, derivatives of thyroxine hormone and ghrelin [10].

But, we chose the administration of 5'-AMP since it is universally available and used by every cell in the body and it has a central role in regulating many signaling pathways. While the ischemia specific markers such as lipocalin-2, cystatin-C, galectin-3 and mucin-1 (MUC-1) are up regulated in animals that received ischemia only, these parameters were significantly attenuated in animals receiving 
5'AMP mediated pre-conditioning prior to ischemia. We are aware of the fact that 5'-AMP could function in several capacities, which are diagrammatically represented in Figure 8.

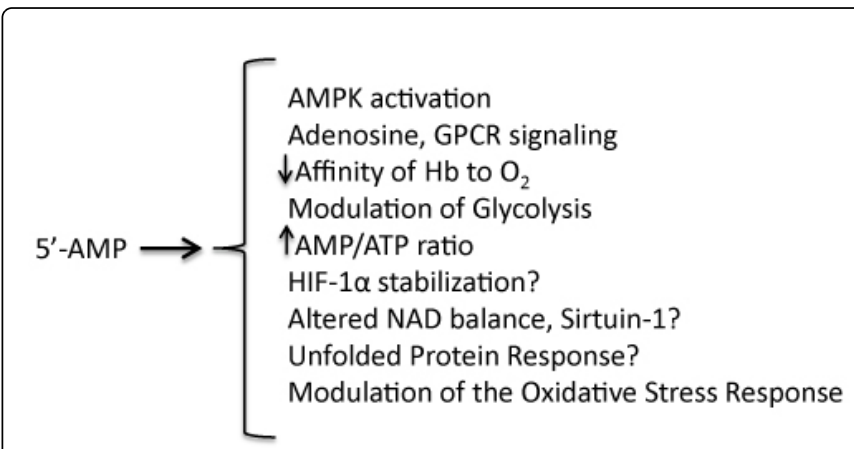

Figure 8: 5'-AMP works through multiple mechanisms. The hypometabolism created by this agent may be the sum of all the individual mechanisms as described in this summary figure. See Discussion section for a detailed explanation of the various branches in the mechanism of action of 5'-AMP.

First, the ratio of AMP to ATP is significantly increased which leads to an imbalance to the energy sensing adenosine nucleotide pool in the body, leading to a slowdown of cellular activity levels. The red blood cells avidly take up this nucleotide since they rely on the activity of the glycolytic pathway as their sole source of energy. Thus, increased intracellular AMP will force the adenylate equilibrium to form ADP utilizing the cellular ATP pool, affecting the ATP-dependent glycolytic pathways [19].

Metabolite characterization and modeling studies have shown that AMP administration leads to an increased accumulation of 2,3 bisphosphoglycerate which decreases the affinity of hemoglobin to oxygen $[19,20]$. Thus, when these oxygen deficient red blood cells circulate through the body, they very likely induce an artificial state of intra-tissue hypoxia, at the least in the case of the kidney, where there is a significant increase in the level of the hypoxia marker protein HIF-1a. Second, it is still theoretically possible that AMP could be dephosphorylated to adenosine in vivo which could then activate the adenosine receptors leading to a decreased cardiac output and hence body temperature. But, among the pathways regulated by 5 '-AMP, the role of the AMP-activated kinase (AMPK) is of paramount importance. Our studies strongly suggest that AMPK is indeed activated and functional in inhibiting the Acetyl CoA carboxylase (ACC) by phosphorylation.

We propose that most of the major effects seen in this study could be explained by the activation of the AMP Kinase. Third, our results characteristically show an up regulation of HIF-1a in the AMPpreconditioned animals. This phenomenon suggests that HIF-1a stabilization could be one of the mechanisms by which AMP elicits its reno-protective effect. This type of hypoxic pre-conditioning has been shown to produce renoprotective effects by earlier studies [26]. The results presented here parallel the studies published earlier by our own laboratory [21-25].

Fourth, we also observe a significant elevation in the level of the anti-oxidant transcription factor Nrf2 as a result of AMP treatment. This can be explained by the fact that AMPK is not only an energy responsive enzyme but also a redox state responsive enzyme
[34-36,41]. Our studies presented here highly suggest that the generation of reactive oxygen and nitrogen species are greatly reduced in ischemic animals pretreated with 5'-AMP are in line with the known mechanistic link between AMPK activation and reduced oxidative stress $[41,42]$.

It is also known that Nrf2 plays a protective role in experimental acute kidney injury (AKI) and this protection is mediated by the endogenous anti-oxidant pathway which is activated by phosphoAMPK $[36,42]$. Thus, induction of the hypometabolic pathway by 5 'AMP may upregulate the anti-oxidant response pathway protecting the kidney tissue through the action of AMPK $[41,42]$. Our studies also indicate the possibility that this hypometabolism inducing approach could be used to extend the warm ischemia time (WIT) while at the same time reduce the reperfusion damage. Our laboratory has been interested in devising several strategies to extend WIT while at the same time preserving global parenchymal function of the kidney [21-25]. This study extends these approaches and highlights some of the potential features that could be exploited for great clinical benefit. For example, on the basis of our animal studies, there could be a " 2 hour window of opportunity" after injecting the prescribed dose of 5'AMP in these non-hibernating mice (Figure 1), wherein there is a marked reduction in both the core body temperature as well as oxygen consumption rate and heart rate. Since the heart's pumping rate was significantly reduced, we interpret that there is a decreased blood flow to the organs, particularly the kidney.

We propose that this could be the window of time wherein a renal artery occlusion surgery could be performed without any appreciable damage to the renal tubules as there was a significant reduction in the oxidative stress, as seen in our studies. It should be noted here that the current window of opportunity to perform renal artery occlusion in partial nephrectomy surgeries with minimum ischemic damage is approximately 30 minutes $[43,44]$. Finally, a potentially significant advantage of this hypometabolism inducing strategy is that the animal comes back to normal state in a "gradual fashion" over a period of time, in which the "reperfusion" process occurs in an incremental and progressive manner.

This could potentially limit the oxidative stress induced damage to the kidneys, as opposed to an "abrupt" reperfusion seen clinically in renal reconstruction surgeries, upon opening the renal pedicle immediately after the surgery, causing severe reperfusion injury to the kidneys. Whether this phenomenon of hypometabolism could be exploited in human beings is still a matter of investigation. It is relevant to note here that mammals evolutionarily close to man, such as the Malagasy primate Cheirogaleus Medius are able to hibernate [45].

Thus, these hibernation and organ protection mechanisms are only beginning to be understood. Apart from the beneficial effects of hypometabolism in protecting the kidneys from ischemia-reperfusion damage, this strategy could also be of great potential benefit in transplantation medicine where organ preservation is of paramount importance for the success of the procedure $[11,12]$.

\section{Acknowledgements}

This study was supported in part from the Susan Schott Memorial Fund and the Saint Vincents Urology Research Fund. Initial discussions with Kenneth B. Storey (Institute of Biochemistry and Department of Biology, Carleton University, Ottawa, Canada) and Zhaoyang Zhao (Departments of Biochemistry and Molecular Biology, University of Texas Health Medical School, Houston, Texas) are 
gratefully acknowledged. The authors are thankful to Bhuvaneswari Dorai for a blinded pathological interpretation of the acute tubular necrosis in the immunohistochemistry slides. The authors also thank John T. Pinto (Biochemistry and Molecular Biology Department, NYMC) for his suggestions and for a careful reading of the manuscript.

\section{References}

1. Geiser F (2013) Hibernation. Curr Biol 23: 188-193.

2. Storey KB, Storey JM (2010) Metabolic rate depression: the biochemistry of mammalian hibernation. Adv Clin Chem 52: 77-108.

3. Storey KB (2010) out cold: biochemical regulation of mammalian hibernation- A mini review. Gerontology 56: 220-230.

4. Andrews MT (2007) Advances in molecular biology of hibernation in mammals. Bio Essays 29: 431-440.

5. Morin P Jr, Storey KB (2009) Mammalian hibernation: differential gene expression and novel application of epigenetic controls. Int J Dev Biol 53 : 433-442.

6. Heldmaier G, Ortmann S, Elvert R (2004) Natural hypometabolism during hibernation and daily torpor in mammals. Respir Physiol Neurobiol 141: 317-329.

7. Heldmaier G (2011) Physiology: Life on low flame in hibernation. Science 331: 866-867.

8. Zhang J, Kaasik K, Blackburn MR, Lee CC (2006) Constant darkness is a circadian metabolic signal in mammals. Nature 439: 340-343.

9. Lee CC (2008) Is human hibernation possible? Annu Rev Med 59: 177-186.

10. Bouma HR, Verhaag EM, Otis JP, Heldmaier G, Swoap SJ, et al. (2012) Induction of torpor: mimicking natural metabolic suppression for biomedical applications. J Cell Physiol 227: 1285-1290.

11. Bernhardt WM, Warnecke C, Willam C, Tanaka T, Wiesener MS, et al. (2007) Organ protection by hypoxia and hypoxia inducible factors. Methods Enzymol 435: 221-245.

12. Guibert EE, Petrenko AY, Balaban CL, Somov AY, Rodriguez JV, et al. (2011) Organ preservation: Current concepts and new strategies for the next decade. Transfus Med Hemother 38: 125-142.

13. Tripatara P, Patel NS, Collino M, Gallichio M, Kieswich J, et al. (2008) Generation of endogenous hydrogen sulfide by cystathionine -gamma lyase limits renal tissue ischemia/reperfusion injury and dysfunction. Lab Invest 88: 1038-1048.

14. Lee HJ, Mariappan MM, Feliers D, Cavaglieri RC, Sataranatarajan K, et al. (2012) Hydrogen sulfide inhibits high glucose-induced matrix protein synthesis by activating AMP-activated protein kinase in renal epithelial cells. J Biol Chem 287: 4451-4461.

15. Bos EM, Leuvenink HG, Snijder PM, Kloosterhuis NJ, Hillebrands JL, et al. (2009) Hydrogen sulfide induced hypometabolism prevents renal ischemia reperfusion injury. J Am Soc Nephrol 20: 1901-1905.

16. Liu YH, Lu M, Bian JS (2011) Hydrogen sulfide and renal ischemia. Expert Rev Clin Pharmacol 4: 49-61.

17. Nicholson CK, Calvert JW (2010) Hydrogen sulfide and ischemiareperfusion injury. Pharmacol Res 62: 289-297.

18. Zhao Z, Miki T, Von Oort-Jansen A, Matsumoto T, Loose DS, et al. (2011) Hepatic gene expression profiling of 5'-AMP induced hypometabolism in mice. Physiol Genomics 43: 325-345.

19. Daniels IS, Zhang J, O’Brien III WG, Tao Z, Miki T, et al. (2010) A role of erythrocytes in adenosine monophosphate initiation of hypometabolism in mammals. J Biol Chem 285: 20716-20723.

20. Zhao Z, Von Oort A, Tao Z, O’Brien III WG, Lee CC, et al. (2014) Metabolite profiling of 5'-AMP induced hypometabolism. Metabolomics 10: 63-76.

21. Michels C, Dorai T, Chander P, Choudhury M, Grasso M, et al. (2009) Hypoxic pre-conditioning in a rat renal ischemia model: an evaluation of the use of hydralazine. World J Urol 27: 817- 823.
22. Dorai T, Michels C, Goldfarb DS, Grasso M (2009) Strategies to extend warm ischemia time during laparoscopic partial nephrectomy-Part I. Int J Nephrol Urol 1: 79-93.

23. Dorai T, Michels C, Goldfarb DS, Grasso M (2010) Strategies to extend warm ischemia time during laparoscopic partial nephrectomy-Part II.Int J Nephrol Urol 2: 157-171.

24. Dorai T, Fishman AI, Ding C, Batinic-Haberle I, Goldfarb DS, et al. (2011) Amelioration of renal ischemia-reperfusion injury with a novel protective cocktail. J Urol 186: 2448-2454.

25. Cohen J, Dorai T, Ding C, Batinic-Haberle I, Grasso M, et al. (2013) Further studies on the extension of warm ischemia time in laparoscopic partial nephrectomy with a novel renoprotective cocktail. J Endourology 27: 343-348.

26. Bernhardt WM, Campean V, Kany S, Jurgensen JS, Wiedemann A, et al. (2006) Preconditional activation of hypoxia inducible factors ameliorates ischemic acute renal failure. J Am Soc Nephrol 17: 1970-1978.

27. Bonventre JV, Yang L (2011) Cellular pathophysiology of ischemic acute kidney injury. J Clin Invest 121: 4210-4221.

28. Fernandez-Bertocchi AP, Campanhole G, Wang PH, Goncalves GM, Damiao MJ, et al. (2008) A role for galectin-3 in renal tissue damage triggered by ischemia and reperfusion injury. Transplant Int 21: 999-1007.

29. Aubert S, Faquette V, Hemon B, Lepoivre R, Briez N, et al. (2009) MUC1 a new hypoxia inducible factor target gene is an actor in clear renal cell carcinoma tumor progression. Cancer Res 69: 5707-5715.

30. Schmidt-Ott KM, Mori K, Li JY, Kalandadze A, Cohen DJ, et al. (2007) Dual action of neutrophil gelatinase-associated lipocalin. J Am Soc Nephrol 18: 407-413.

31. Song S, Meyer M, Turk TR, Wilde B, Feldkamp T, et al. (2009) Serum cystatin- $\mathrm{C}$ in mouse models: a reliable and precise marker for renal function and superior to serum creatinine. Nephrol Dial Transplant 24: 1157-1161.

32. Ramnanen CJ, McMullen DC, Groom AG, Storey KB (2010) The regulation of AMPK signaling in a natural state of profound metabolic rate depression. Mol Cell Biochem 335: 91-105.

33. Calvani M, Comito G, Giannoni E, Chiarugi P (2012) Time dependent stabilization of hypoxia inducible factor-1a by different intracellular sources of reactive oxygen species. PLoS One 7: e38388.

34. Hardie DG, Ross FA, Hawley SA (2012) AMPK: a nutrient and energy sensor that maintains energy homeostasis. Nat Rev Mol Cell Biol 13: 251-262.

35. Hardie DG (2011) AMP-activated protein kinase-an energy sensor that regulates all aspects of cell function. Genes Dev 25: 1895-1908.

36. Leonard MO, Kieran NE, Howell K, Burne MJ, Varadarajan R, et al. (2006) Re-oxygenation-specific activation of the antioxidant transcription factor Nrf2 mediates cytoprotective gene expression in ischemiareperfusion injury. FASEB J 20: 2624-2626.

37. Horman S, Hussain N, Dilworth SM, Storey KB, Rider MH, et al. (2005) Evaluation of the role of AMP activated protein kinase and its downstream targets in mammalian hibernation. Comp Biochem Physiol B Biochem Mol Biol 142: 374-382.

38. Liu M, Grigoryev DN, Crow MT, Haas M, Yamamoto M, et al. (2009) Transcription factor Nrf2 is protective during ischemic and nephrotoxic acute kidney injury in mice. Kidney Int 76: 277-285.

39. Jani A, Martin SL, Jain S, Keys D, Edelstein CL, et al. (2013) Renal adaptation during hibernation. Am J Physiol Renal Physiol 305: 1521-1532.

40. Jani A, Epperson E, Martin J, Pacic A, Ljubanovic D, et al. (2011) Renal protection from prolonged cold ischemia and warm reperfusion in hibernating squirrels. Transplantation 92: 1215-1221.

41. Cardaci S, Filomeni G, Ciriolo MR (2012) Redox implications of AMPKmediated signal transduction beyond energetic clues. J Cell Sci 125: 2115-2125.

42. Mo C, Wang L, Zhang J, Numazawa S, Tang H, et al. (2014) The crosstalk between Nrf2 and AMPK signal pathways is important for the anti- 
Citation: $\quad$ Alexander B, James M, Fishman A, Grasso M, Dorai T (2016) Induction of Hypometabolism as a Novel Strategy to Minimize Ischemia Reperfusion Injury in Renal Reconstruction Surgeries. J Transplant Technol Res 6: 164. doi:10.4172/2161-0991.1000164

Page 9 of 9

inflammatory effect of berberine in LPS-stimulated macrophages and endotoxin shocked mice. Antioxid Redox Signal 20: 574-588.

43. Porpiglia F, Renard J, Billia M, Musso F, Volpe A, et al. (2007) Is renal warm ischemia over 30 minutes during laparoscopic partial nephrectomy possible? One year results of a prospective study. Eur Urol 52: 1170-1178.
44. Janetschek G (2007) Laparoscopic partial nephrectomy for RCC: How can we avoid ischemic damage of the renal parenchyma? Eur Urol 52: 1303-1305.

45. Dausmann KH, Gos J, Ganzhom JU, Heldmaier G (2005) Hibernation in the tropics: Lessons from a primate. J Comp Physiol B 175: 147-155. 